\title{
TYPES OF COMPLETE INFINITELY SHEETED PLANES
}

\author{
MITSURU NAKAI
}

\begin{abstract}
We will answer negatively to the question whether the completeness of infinitely sheeted covering surfaces of the extended complex plane have anything to do with their types being parabolic or hyperbolic. This will be accomplished by giving a one parameter family $\{W[\alpha]: \alpha \in \mathcal{A}\}$ of complete infinitely sheeted planes $W[\alpha]$ depending on the parameter set $\mathcal{A}$ of sequences $\alpha=\left(a_{n}\right)_{n \geq 1}$ of real numbers $0<a_{n} \leq 1 / 2(n \geq 1)$ such that $W[\alpha]$ is parabolic for 'small' $\alpha$ 's and hyperbolic for 'large' $\alpha$ 's.
\end{abstract}

\section{$\S 1$. Introduction}

The purpose of this paper is to show the existence of a one parameter family $(W[\alpha])_{\alpha \in \mathcal{A}}$ of complete infinitely sheeted planes $W[\alpha]$ such that $W[\alpha] \in O_{G}$ for 'small' $\alpha \in \mathcal{A}$ and $W[\alpha] \notin O_{G}$ for 'large' $\alpha \in \mathcal{A}$.

Recall that a covering (Riemann) surface $X$, or more precisely, $(X, Y, f)$ of $Y$ is a triple of two Riemann surfaces $X$ and $Y$ and an analytic mapping $f$ of $X$ to $Y$. It is said to be complete (cf. [2]) if every $a \in Y$ has a closed parametric disc $K$ around $a$ such that each component of $f^{-1}(K)$ is compact. When $(X, Y, f)$ is smooth, i.e. there is no branch point in $X$, the completeness of $(X, Y, f)$ is equivalent to the regularity of $(X, Y, f)$, where $(X, Y, f)$ is regular if, for any $\operatorname{arc} \gamma$ on $Y$ and any point $\tilde{a} \in X$ lying over the initial point $a$ of $\gamma$, there always exists a continuation $\tilde{\gamma}$ on $X$ along $\gamma$ starting from $\tilde{a}$, i.e. there is an $\operatorname{arc} \tilde{\gamma}$ on $X$ with its initial point $\tilde{a}$ such that $f(\tilde{\gamma})=\gamma($ cf. $[2])$.

Let $P$ be the plane, or more precisely, $P$ be the finite complex plane $\mathbf{C}:|z|<\infty$ or the infinite (i.e. extended) complex plane $\widehat{\mathbf{C}}:|z| \leq+\infty$ (i.e. $\widehat{\mathbf{C}}=\mathbf{C} \cup\{\infty\}$, the Riemann sphere). If the covering surface $(X, P, \pi)$ of $P$ satisfies the following two conditions, then $X$, or $(X, P, \pi)$, is referred to as a multisheeted plane: the cardinal number card $\pi^{-1}(a)$ is a constant

Received March 11, 2003.

2000 Mathematics Subject Classification: Primary 30F20, 30F25; Secondary 30C25, $30 \mathrm{C} 35$. 
$\mu_{X} \in \mathbf{N} \cup\left\{\aleph_{0}\right\}$ for every $a \in P$, where $\mathbf{N}$ is the set of positive integers and $\aleph_{0}=$ card $\mathbf{N}$; for any cover continuation $\left(X^{\prime}, P, \pi^{\prime}\right)$ of $(X, P, \pi)$, the closed set $X^{\prime} \backslash X$ is of logarithmic capacity zero measured on $X^{\prime}$, where a cover continuation $\left(X^{\prime}, P, \pi^{\prime}\right)$ of $(X, P, \pi)$ is a covering surface of $P$ such that $X$ is a subsurface of $X^{\prime}$ such that $\pi^{\prime} \mid X=\pi$. If the number, which we call the sheet number of $X, \mu_{X} \in \mathbf{N}\left(\mu_{X}=\aleph_{0}\right.$, resp. $)$, then $(X, P, \pi)$ is said to be a finitely (infinitely, resp.) sheeted plane.

What we call our type problem is to judge whether $X \in O_{G}$ or not for a given multisheeted plane $(X, P, \pi)$ (cf. [7], [11]), where $O_{G}$ is the class of parabolic (i.e. not hyperbolic) Riemann surfaces characterized by the nonexistence of Green function on them (cf. e.g. [8], [9]). If $(X, P, \pi)$ is finitely sheeted plane, then we can easily see that $X \in O_{G}$ along with the parabolicity or the ellipticity of $P$. In view of this, we will consider only infinitely sheeted planes in our type problem. We have been having a feeling that complete infinitely sheeted planes are apt to become parabolic. In fact, we have shown the existence of a family $\mathcal{W}$ of infinitely sheeted planes $(X, \mathbf{C}, \pi)$ which is an equivalence class of infinitely sheeted planes with respect to the similarity, where $\left(X_{1}, \mathbf{C}, \pi_{1}\right)$ and $\left(X_{2}, \mathbf{C}, \pi_{2}\right)$ are similar to each other if there is a topological mapping $T$ of $X_{1}$ onto $X_{2}$ with the property that $p \in X_{1}$ is a branch point of multiplicity $m$ if and only if $T(p) \in X_{2}$ is a branch point of multiplicity $m$ and in this case $\pi_{1}(p)=$ $\pi_{2}(T(p))$, such that $X \in O_{G}$ for a $(X, \mathbf{C}, \pi) \in \mathcal{W}$ if and only if $(X, \mathbf{C}, \pi)$ is complete (cf. [5] and [6]). Nevertheless, the main purpose of this paper is to maintain that the parabolicity and the completeness of infinitely sheeted planes have absolutely nothing to do with each other. This will be shown by constructing a one sequential parameter family $(W[\alpha])_{\alpha \in \mathcal{A}}$ of complete infinitely sheeted planes $(W[\alpha], \widehat{\mathbf{C}}, \pi)(\alpha \in \mathcal{A})$ such that $W[\alpha] \in O_{G}$ if $\alpha$ is "small" and $W[\alpha] \notin O_{G}$ if $\alpha$ is "large", where $\mathcal{A}$ is the family of sequences $\alpha=\left(a_{n}\right)_{n \in \mathbf{N}}$ with $0<a_{n} \leq 1 / 2$. The one sequential parameter family $\left(W[\alpha] \backslash \pi^{-1}(\infty)\right)_{\alpha \in \mathcal{A}}$ of $\left(W[\alpha] \backslash \pi^{-1}(\infty), \mathbf{C}, \pi\right)(\alpha \in \mathcal{A})$ plays the same role as that of $(W[\alpha])_{\alpha \in \mathcal{A}}$.

\section{$\S 2$. Construction of a one parameter family}

Consider the class $\mathcal{A}$ of sequences $\alpha=\left(a_{n}\right)_{n \in \mathbf{N}}$ of real numbers $a_{n}$ with $0<a_{n} \leq 1 / 2(n \in \mathbf{N})$. We view $\mathcal{A}=\{\alpha\}$ the sequential parameters family. We will construct a complete infinitely sheeted plane $(W[\alpha], \widehat{\mathbf{C}}, \pi)$ for each $\alpha \in \mathcal{A}$ so that we obtain a one sequential parameter family $(W[\alpha])_{\alpha \in \mathcal{A}}$ of complete infinitely sheeted planes $W[\alpha]$. 
Let $J=[0,1 / 2]$ be the slit in $\widehat{\mathbf{C}}$. We denote by $S$ the surface obtained from two copies of $\widehat{\mathbf{C}} \backslash J$ by pasting $\widehat{\mathbf{C}} \backslash J$ and $\widehat{\mathbf{C}} \backslash J$ crosswise along $J$. The closure of one of $\widehat{\mathbf{C}} \backslash J$ in $S$ will be called the upper sheet of $S$ and denoted by $S^{+}$. The other closure of $\widehat{\mathbf{C}} \backslash J$ left in $S$ is then referred to as the lower sheet of $S$ and denoted by $S^{-}$. The surface $S$ is conformally the sphere $\widehat{\mathbf{C}}$ but presently represented as a two-sheeted plane.

In addition to $\mathcal{A}$ and $S$ we use the index set $\Lambda=\{-2,-1,1,2\}$ as another ingredient in our construction of $W[\alpha](\alpha \in \mathcal{A})$. An admissible $n$-tuple $i_{1} i_{2} \cdots i_{n}$ of elements in $\Lambda$ is an ordered $n$-tuple of $i_{1}, i_{2}, \ldots, i_{n}$ in $\Lambda$ such that $i_{k} \neq-i_{k-1}(k=2, \ldots, n)$. There are $4=4 \cdot 3^{1-1}$ admissible 1-tuples $i_{1}$ in $\Lambda, 4 \cdot 3=4 \cdot 3^{2-1}$ admissible 2-tuples $i_{1} i_{2}, 4 \cdot 3^{2}=4 \cdot 3^{3-1}$ admissible 3 -tuples $i_{1} i_{2} i_{3}$, and so on, ... and in general $4 \cdot 3^{n-1}$ admissible $n$-tuples $i_{1} \cdots i_{n}$.

First consider 4 slits $I_{0 i}=[|i|,|i|+1 / 2](i \in \Lambda)$ in $S$ by the following rule: $I_{0 i} \subset S^{+}$for $i>0$ and $I_{0 i} \subset S^{-}$for $i<0$ so that $I_{01}$ and $I_{02}$ are in $S^{+}$and $I_{0,-1}$ and $I_{0,-2}$ are in $S^{-}$. Then we consider the 4-slitted $S$ :

$$
F_{0}:=S \backslash \bigcup_{i \in \Lambda} I_{0 i}
$$

We now fix an $\alpha=\left(a_{n}\right)_{n \in \mathbf{N}} \in \mathcal{A}$ so that $0<a_{n} \leq 1 / 2(n \in \mathbf{N})$. Let $I_{n i}:=\left[|i|,|i|+a_{n}\right]$ and

$$
F_{i_{1} \cdots i_{n}}:=S \backslash\left(I_{n-1, i_{n}} \cup\left(\bigcup_{i \in \Lambda \backslash i_{n}} I_{n i}\right)\right)
$$

for each $i_{1} \cdots i_{n}$ of $4 \cdot 3^{n-1}$ admissible $n$-tuples in $\Lambda(n \in \mathbf{N})$. We then form an infinitely sheeted planes $W[\alpha]$, which we express symbolically as follows:

$$
W[\alpha]:=F_{0}+\sum_{i_{1}} F_{i_{1}}+\sum_{i_{1} i_{2}} F_{i_{1} i_{2}}+\cdots+\sum_{i_{1} \cdots i_{n}} F_{i_{1} \cdots i_{n}}+\cdots,
$$

where $i_{1} \cdots i_{n}$ are admissible $n$-tuples $(n \in \mathbf{N})$. Here we add a few words to mention the exact procedure in the construction in (3). As the first step, we perform the following: each $F_{i_{1}}$ of the four $F_{i_{1}}\left(i_{1} \in \Lambda\right)$ is pasted to $F_{0}$ crosswise along $I_{0,-i_{1}}$ in $F_{0}$ and $I_{0 i_{1}}$ in $F_{i_{1}}$. From the second step on, and in general as the $n^{\text {th }}$ step, we perform the following: each $F_{i_{1} \cdots i_{n-1} i_{n}}$ of three $F_{i_{1} \cdots i_{n-1} i_{n}}\left(i_{n} \in \Lambda \backslash\left\{-i_{n-1}\right\}\right)$ is pasted to each $F_{i_{1} \cdots i_{n-1}}$ of $4 \cdot 3^{n-2} F_{i_{1} \cdots i_{n-1}}$ for every admissible $(n-1)$-tuple $i_{1} \cdots i_{n-1}$ in $\Lambda$ crosswise along $I_{n-1,-i_{n}}$ in $F_{i_{1} \cdots i_{n-1}}$ and $I_{n-1, i_{n}}$ in $F_{i_{1} \cdots i_{n-1} i_{n}}$. 
From the construction it is clear that each $p \in W[\alpha]$ lies above a point in $\widehat{\mathbf{C}}$, which we denote by $\pi(p)$. Then $p \mapsto \pi(p)$ is an analytic mapping of $W[\alpha]$ to $\widehat{\mathbf{C}}$. Now it is easy to see that $W[\alpha]$, or more precisely $(W[\alpha], \widehat{\mathbf{C}}, \pi)$, is a complete infinitely sheeted plane. Moreover $W[\alpha]$ is planar. Concerning the one parameter family $(W[\alpha])_{\alpha \in \mathcal{A}}$ constructed above, we have the following result.

TheOrem. The complete infinitely sheeted plane $W[\alpha]$ determined by the sequence $\alpha=\left(a_{n}\right)_{n \in \mathbf{N}}$ with $0<a_{n} \leq 1 / 2(n \in \mathbf{N})$ is hyperbolic, i.e. $W[\alpha] \notin O_{G}$, if $\alpha$ is large in the sense that

$$
\inf _{n \in \mathbf{N}} a_{n}>0
$$

and $W[\alpha]$ is parabolic, i.e. $W[\alpha] \in O_{G}$, if $\alpha$ is small in the sense that

$$
\sum_{n \in \mathbf{N}} \frac{1}{3^{n}} \log \frac{1}{a_{n}}=+\infty
$$

The proof of the second part of our theorem above will be given in the next Section 3 and the first part in Section 4. The proof of the second part is easy and very short. Since the proof given in Section 4 is considerably long, the section is divided into 3 Subsections 4.1-4.3.

\section{§3. Proof of Theorem: parabolicity}

We now start the proof of our theorem stated in Section 2. In this present short Section 3 we prove that if $\alpha=\left(a_{n}\right)_{n \in \mathbf{N}} \in \mathcal{A}$ is so small as to satisfy (5), then $W[\alpha] \in O_{G}$. For simplicity we set $W:=W[\alpha]$. Let $W_{n}$ $(n \geq 0)$ be regular subregions of $W$ given by

(6) $W_{0}:=F_{0}, \quad W_{n}:=F_{0}+\sum_{i_{1}} F_{i_{1}}+\sum_{i_{1} i_{2}} F_{i_{1} i_{2}}+\cdots+\sum_{i_{1} \cdots i_{n}} F_{i_{1} \cdots i_{n}}(n \in \mathbf{N})$,

where $i_{1} \cdots i_{k}(1 \leq k \leq n)$ are admissible $k$-tuples $(1 \leq k \leq n)$. Then $\left(W_{n}\right)_{n \geq 0}$ forms a regular exhaustion of $W$, which is called the standard exhaustion of $W$ in this present paper. For each $n \in \mathbf{N}$ let $w_{n}$ be the continuous function on $\bar{W}_{n} \backslash W_{n-1}$ such that $w_{n}$ is harmonic on $W_{n} \backslash \bar{W}_{n-1}$ with boundary values $w_{n} \mid \partial W_{n}=1$ and $w_{n} \mid \partial W_{n-1}=0$. The function $w_{n}$ is referred to as the harmonic measure of $\partial W_{n}$ on $W_{n} \backslash \bar{W}_{n-1}$. The Dirichlet integral $D_{W_{n} \backslash \bar{W}_{n-1}}\left(w_{n}\right)$ of $w_{n}$ over $W_{n} \backslash \bar{W}_{n-1}$ is, by definition,

$$
D_{W_{n} \backslash \bar{W}_{n-1}}\left(w_{n}\right):=\int_{W_{n} \backslash \bar{W}_{n-1}}\left|\nabla w_{n}(z)\right|^{2} d x d y \quad(z=x+\sqrt{-1} y) .
$$


Then the modulus $\mu_{n}$ of the configuration $W_{n} \backslash \bar{W}_{n-1}$ is given by

$$
\mu_{n}:=2 \pi / D_{W_{n} \backslash \bar{W}_{n-1}}\left(w_{n}\right) .
$$

By virtue of (5) we will derive

$$
\sum_{n \in \mathbf{N}} \mu_{n}=+\infty
$$

Before proceeding to the proof of the above (7), we pause here to make the following simple observation. We denote by $A_{a}$ for $0<a \leq 1 / 4$ the annulus bounded by the ellipse centered at the origin 0 with major axis 1 and minor axis $\sqrt{1-4 a^{2}}$ and the segment $[-a, a]$, by $B_{a}$ the annulus bounded by the ellipse centered at 0 with major axis $1 / a$ and minor axis $\sqrt{1-4 a^{2}} / a$ and the segment $[-1,1]$, and finally by $C_{a}$ the ring domain bounded by circles $|z|=2 a /\left(1+\sqrt{1-4 a^{2}}\right)$ and $|z|=1$. By the conformal mapping $w=(z+1 / z) / 2, C_{a}$ is mapped onto $B_{a}$. By the conformal mapping $w=a z, B_{a}$ is mapped onto $A_{a}$. In view of $\bmod A_{a}=\bmod B_{a}=\bmod C_{a}$, where e.g. $\bmod A_{a}$ means the modulus of $A_{a}$, and

$$
\bmod C_{a}=\log \left[\left(1+\sqrt{1-4 a^{2}}\right) / 2 a\right]
$$

we see that

$$
\bmod A_{a}=\log \left[\left(1+\sqrt{1-4 a^{2}}\right) / 2 a\right]=2 \pi / D_{A_{n}}(w),
$$

where $w$ is the harmonic measure of the segment $[-a, a]$ on $A_{a}$. From the above (8) it can be derived that

$$
\frac{1}{2} \log \frac{1}{a} \leq \bmod A_{a} \leq \log \frac{1}{a} .
$$

We now turn to the proof of (7). Observe that $W_{n} \backslash \bar{W}_{n-1}$ consists of $4 \cdot 3^{n-1}$ components $F_{i_{1} \cdots i_{n}}$ for all admissible $n$-tuples $i_{1} \cdots i_{n}$. Since the modulus $\mu_{i_{1} \cdots i_{n}}$ of $F_{i_{1} \cdots i_{n}}$ is given by $2 \pi / D_{F_{i_{1} \cdots i_{n}}}\left(w_{n}\right)$, where $w_{n}$ is the harmonic measure of $\partial W_{n}$ on $W_{n} \backslash \bar{W}_{n-1}$, and

$$
D_{W_{n} \backslash \bar{W}_{n-1}}\left(w_{n}\right)=\sum_{i_{1} \cdots i_{n}} D_{F_{i_{1} \cdots i_{n}}}\left(w_{n}\right),
$$

we conclude that

$$
1 / \mu_{n}=\sum_{i_{1} \cdots i_{n}} 1 / \mu_{i_{1} \cdots i_{n}}
$$


Let $A:=A_{a_{n-1} / 2}+\left(\left|i_{n}\right|+a_{n-1} / 2\right)$, where $a_{0}=1 / 2$, and $w$ be the harmonic measure of the segment part of $\partial A$ on $A$. Since $A \subset F_{i_{1} \cdots i_{n}}$, the Dirichlet principle implies

$$
D_{A}(w)=D_{F_{i_{1} \cdots i_{n}}}(w) \geq D_{F_{i_{1} \cdots i_{n}}}\left(w_{n}\right),
$$

where $w$ is extended to $F_{i_{1} \cdots i_{n}}$ from $A$ by setting $w=0$ on $F_{i_{1} \cdots i_{n}} \backslash A$. Thus $\bmod A_{a_{n-1} / 2}=\bmod A \leq \mu_{i_{1} \cdots i_{n}}$. Using (10) we see that

$$
1 / \mu_{n} \leq \sum_{i_{1} \cdots i_{n}} 1 /\left(\frac{1}{2} \log \frac{1}{a_{n-1}}\right)=4 \cdot 3^{n-1} /\left(\frac{1}{2} \log \frac{1}{a_{n-1}}\right)
$$

or $\mu_{n} \geq 8^{-1} 3^{-(n-1)} \log \left(1 / a_{n-1}\right)$. Therefore (5) implies

$$
\sum_{n \in \mathbf{N}} \mu_{n} \geq 8^{-1} \sum_{n \in \mathbf{N}} 3^{-(n-1)} \log \left(1 / a_{n-1}\right)=+\infty,
$$

which is the relation (7) to be proved.

By the Sario-Noshiro modular criterion for the parabolicity of $W$ (cf. e.g. [8], [9], etc.), the existence of the regular exhaustion $\left(W_{n}\right)_{n \in \mathbf{N}}$, which we call the standard exhaustion of $W$ in this paper, of $W$ with (7) concludes that $W \in O_{G}$.

\section{§4. Proof of Theorem: hyperbolicity}

Under the assumption that the sequence $\alpha=\left(a_{n}\right)_{n \in \mathbf{N}}$ is large in the sense that $c:=\inf _{n \in \mathbf{N}} a_{n}>0$, we will show that $W[\alpha]$ is hyperbolic, i.e. $W[\alpha] \notin O_{G}$. As the largest member in $\mathcal{A}$ will be referred to the sequence $\sigma:=\left(s_{n}\right)_{n \in \mathbf{N}}$ such that $s_{n}=1 / 2(n \in \mathbf{N})$ so that

$$
\sigma=(1 / 2,1 / 2, \ldots, 1 / 2, \ldots) .
$$

The plan of our proof for $W[\alpha] \notin O_{G}$ goes as follows. First we will show in Subsection 4.1 the existence of a quasiconformal mapping $T$ of $W[\sigma]$ onto $W[\alpha]$. By the quasiconformal invariance of the parabolicity (i.e. if there is a quasiconformal mapping of a Riemann surface $R_{1}$ onto another $R_{2}$, then $R_{1} \in O_{G}$ if and only if $R_{2} \in O_{G}$ (cf. e.g. [9])), we can conclude our objective $W[\alpha] \notin O_{G}$ if we show that $W[\sigma] \notin O_{G}$. Then, as the second step, we will derive in Subsections 4.2 and 4.3 a contradiction from the erroneous assumption $W[\sigma] \in O_{G}$. 


\subsection{Construction of a quasiconformal mapping}

In this first Subsection 4.1, we will construct a quasiconformal mapping $T$ of $W[\sigma]$ onto $W[\alpha]$. We consider, as we did in Section 3, the standard exhaustions for covering surfaces constructed in Section 2. Recall that the standard exhaustion $\left(W_{n}[\alpha]\right)_{n \geq 0}$ of $W[\alpha]$ is given by

$$
W_{0}[\alpha]:=F_{0}, \quad W_{n}[\alpha]:=F_{0}+\sum_{i_{1}} F_{i_{1}}+\cdots+\sum_{i_{1} \cdots i_{n}} F_{i_{1} \cdots i_{n}}(n \geq 1),
$$

where $F_{i_{1} \cdots i_{k}}$ will also be denoted by $F_{i_{1} \cdots i_{k}}[\alpha]$ indicating the dependence on $\alpha$. Take a standard neighborhood $U_{n}[\alpha]$ of each $\partial W_{n}[\alpha](n \in \mathbf{N})$ characterized by the following three conditions: a) each component of $U_{n}[\alpha]$ is a piecewise smooth annulus containing only one component of $\partial W_{n}[\alpha]$, where a region is piecewise smooth if the region is relatively compact and its relative boundary consists of a finite number of mutually disjoint piecewise smooth Jordan curves; b) any two different components of $\bigcup_{n>0} U_{n}[\alpha]$ are disjoint in their closures; c) $W[\alpha] \backslash \bigcup_{n \geq 0} \overline{U_{n}[\alpha]}$ is an open set independent of $\alpha$ so that, for example,

$$
W[\alpha] \backslash \bigcup_{n \geq 0} \overline{U_{n}[\alpha]}=W[\sigma] \backslash \bigcup_{n \geq 0} \overline{U_{n}[\sigma]} .
$$

Let $\gamma[\alpha]$ be a Jordan curve which is a component of $\partial W_{n}[\alpha]$ and let $V[\alpha]$ be the annulus containing $\gamma[\alpha]$ which is a component of $U_{n}[\alpha]$.

The construction of $T$ goes as follows. First let the mapping $T$ be defined as the identity between $W[\sigma] \backslash \bigcup_{n \geq 0} U_{n}[\sigma]$ and $W[\alpha] \backslash \bigcup_{n \geq 0} U_{n}[\alpha]$. The construction will be over if we define a $K$-quasiconformal mapping of $V[\sigma]$ onto $V[\alpha]$ for every $V[\alpha]$ and its corresponding $V[\sigma]$ such that $T: \partial V[\sigma] \mapsto \partial V[\alpha]$ is the identity and $K \leq K_{0}$, where $K_{0}$ is a constant independent of the choice of $V[\alpha]$ (and $V[\sigma]$ ).

Suppose $\gamma[\alpha]$ comes from the slit $I_{n, i_{n+1}}=I_{n, i_{n+1}}[\alpha]=\left[\left|i_{n+1}\right|,\left|i_{n+1}\right|+\right.$ $\left.a_{n}\right]$ in $F_{i_{1} \cdots i_{n} i_{n+1}}=F_{i_{1} \cdots i_{n} i_{n+1}}[\alpha]$ and the slit $I_{n,-i_{n+1}}=I_{n,-i_{n+1}}[\alpha]$ in $F_{i_{1} \cdots i_{n}}=F_{i_{1} \cdots i_{n}}[\alpha]$, which are pasted crosswise and give rise to the analytic Jordan curve $\gamma[\alpha]$. Let $\gamma[\sigma]$ be the corresponding one to $\gamma[\alpha]$ in $W[\sigma]$ so that it comes from $I_{n+1, i_{n+1}}[\sigma]=\left[\left|i_{n+1}\right|,\left|i_{n+1}\right|+s_{n}\right]$ in $F_{i_{1} \cdots i_{n} i_{n+1}}[\sigma]$ and $I_{n,-i_{n+1}}[\sigma]=\left[\left|-i_{n+1}\right|,\left|-i_{n+1}\right|+s_{n}\right]$ in $F_{i_{1} \cdots i_{n}}[\sigma]$, which are pasted crosswise and give rise to $\gamma[\sigma]$.

We denote by $Z(d)$ the slitted square

$$
Z(d):=Q \backslash J(d)
$$


where $Q=(0,1) \times(0,1)$ is the unit square and $J(d)=[1 / 4,1 / 4+d]+\sqrt{-1} / 2$ is the line segment of the length $0<d \leq 1 / 2$ and set

$$
Z_{i}(d):=Z(d)-\sqrt{-1} / 2+|i|-1 / 4
$$

for $i \in \Lambda$. Then $Z_{i_{n+1}}\left(a_{n}\right)$ is viewed as being contained in $F_{i_{1} \cdots i_{n} i_{n+1}}[\alpha]$ and the slit of $Z_{i_{n+1}}\left(a_{n}\right)$ is identical with $I_{n, i_{n+1}}[\alpha]$. Similarly $Z_{-i_{n+1}}\left(a_{n}\right)$ is viewed as being contained in $F_{i_{1} \cdots i_{n}}[\alpha]$ and the slit of $Z_{-i_{n+1}}\left(a_{n}\right)$ is identical with $I_{n,-i_{n+1}}[\alpha]$. Thus as $V[\alpha]$ we take the annulus obtained by pasting $Z_{i_{n+1}}\left(a_{n}\right)$ to $Z_{-i_{n+1}}\left(a_{n}\right)$ crosswise along $I_{n, i_{n+1}}$ and $I_{n,-i_{n+1}}$. If we can construct a quasiconformal mapping $T$ of $Z_{i_{n+1}}\left(s_{n}\right) \cup I_{n, i_{n+1}}[\sigma]$ to $Z_{i_{n+1}}\left(a_{n}\right) \cup$ $I_{n, i_{n+1}}[\alpha]$ such that $T$ is identity on their boundaries and

$$
T\left(I_{n, i_{n+1}}[\sigma]\right)=I_{n, i_{n+1}}[\alpha]
$$

then it can be continued symmetrically to the mapping $T$ of $V[\sigma]$ to $V[\alpha]$ with the dilatation less than a fixed constant $K_{0}$ independent of $\gamma[\sigma]$ and $\gamma[\alpha]$.

Based upon the observation above we, hence, only have to show the existence of a quaiconformal mapping $w$ from the square $Q=(0,1) \times(0,1)$ to itself fixing $\partial Q$ such that $w(I(1 / 2))=I\left(a_{n}\right)$, where

$$
I(1 / 2):=[1 / 4,1 / 4+1 / 2]+\sqrt{-1} / 2, \quad I\left(a_{n}\right):=\left[1 / 4,1 / 4+a_{n}\right]+\sqrt{-1} / 2
$$

and the dilatation $K$ is bounded by a constant $K_{0}$ depending only on $c$. For simplicity we set $a_{n}=: d$ in this proof so that $I(d):=I\left(a_{n}\right)$ and $0<c \leq d \leq 1 / 2$.

Consider a homeomorphic mapping $w$ of $Q$ onto itself given by

$$
w(z):=u(z)+\sqrt{-1} y \quad(z=x+\sqrt{-1} y),
$$

where $u(z):=x$ for $0 \leq x \leq 1 / 4,2(\xi(y)-1 / 4)(x-1 / 4)+1 / 4$ for $1 / 4 \leq$ $x \leq 3 / 4$, and $4(1-\xi(y))(x-1)+1$ for $3 / 4 \leq x \leq 1$, where

$$
\xi(y):=(1-2 d)|y-1 / 2|+(1 / 4+d),
$$

which maps $[0,1]$ to $[1 / 4+d, 3 / 4]$. Then $w_{x}=u_{x}$ and $w_{y}=u_{y}+\sqrt{-1}$ and thus $w_{z}=\left(w_{x}-\sqrt{-1} w_{y}\right) / 2=\left(u_{x}+1-\sqrt{-1} u_{y}\right) / 2$ and $w_{\bar{z}}=\left(w_{x}+\right.$ $\left.\sqrt{-1} w_{y}\right) / 2=\left(u_{x}-1+\sqrt{-1} u_{y}\right) / 2$ so that the candidate of complex dilatation

$$
\mu(z):=w_{\bar{z}}(z) / w_{z}(z)
$$


satisfies $|\mu|^{2}=(P-p) /(P+p)$, where $P:=\left(u_{x}^{2}+u_{y}^{2}+1\right) / 2$ and $p:=u_{x}$. Since $P \leq P_{0}:=11 / 2$ and $p \geq p_{0}:=2 d$, we have

$$
|\mu|^{2}=\frac{P-p}{P+p} \leq \frac{P_{0}-p_{0}}{P_{0}+p_{0}}=\left(\frac{K-1}{K+1}\right)^{2}
$$

or equivalently

$$
|\mu| \leq \frac{K-1}{K+1}
$$

where

$$
K:=\frac{11}{4 d}+\sqrt{\frac{121}{16 d^{2}}-1} \leq \frac{11}{4 c}+\sqrt{\frac{121}{16 c^{2}}-1}=: K_{0}
$$

and

$$
K \geq \frac{11}{4 \cdot(1 / 2)}+\sqrt{\frac{121}{16 \cdot(1 / 2)^{2}}-1}=\frac{11}{2}+\sqrt{\frac{1}{4}+29}>1 .
$$

Hence $\mu$ defined above is a measurable function on $\bar{Q}$ such that $|\mu| \leq$ $(K-1) /(K+1)$ on $\bar{Q}$ so that $\mu$ is eligible to be a complex dilatation on $\bar{Q}$. Clearly $w: \bar{Q} \mapsto \bar{Q}$ is a homeomorphism, the identity on $\partial Q$, ACL on $\bar{Q}$ and $w(I(1 / 2))=I(d)$, and satisfies the Beltrami equation

$$
w_{\bar{z}}(z)=\mu(z) w_{z}(z)
$$

almost everywhere on $\bar{Q}$, i.e. $w$ is a generalized solution of the above Beltrami equation on $\bar{Q}$. Therefore $w$ is a required $K$-quasiconformal mapping of $\bar{Q}$ to $\bar{Q}$ (cf. e.g. [3]) fixing $\partial Q$ pointwise with $K \leq K_{0}$, a fixed constant depending only upon $c$.

\subsection{Exhausting regions with short boundaries}

We are in the position to prove $W[\sigma] \notin O_{G}$. We will do this by contradiction so that we now make the erroneous assumption that $W:=$ $W[\sigma] \in O_{G}$. Then there exists an Evans-Selberg potential $h$ on $W$ with its pole at $a \in W$ (cf. [4], see also [9] and [10]): $h$ is a harmonic function on $W \backslash a$ such that $h$ has the negative logarithmic pole at $a \in W$, i.e. $z \mapsto h(z)+\log (1 /|z|)$ is harmonic at $a$ for the local parameter $z$ at $a$ with $z(a)=0$, and $h(z) \rightarrow+\infty$ as $z$ tends to the Alexandroff point of $W$. We consider the polar coordinate $r e^{\sqrt{-1} \theta}$ on $W$ constructed from $h$, where $r=e^{h}$ and $\theta$ is the multivalued conjugate harmonic function of $h$ on $W \backslash a$ so that $d \theta=* d h$ on $W \backslash a$. Using a branch $\theta$ of $\theta$ we can use $r e^{\sqrt{-1} \theta}$ as 
local parameters at each point of $W$ except for an isolated set of points with $|\nabla h|=0$.

For each $0<t<+\infty$ we set

$$
R(t):=\{z \in W: h(z)<\log t\}=\{z \in W: r=r(z)<t\}
$$

so that $a \in R(t)$ since $r(a)=0$. Clearly $R(t)$ is a relatively compact region and its relative boundary $\Gamma(t):=\partial R(t)$ consists of a finite number of Jordan curves which may have common points at where $* d h=0$. Except for these points which are isolated in $W$ the above Jordan curves are analytic. Hence except for a countable number of $0<t<+\infty, R(t)$ is a regular region and $\Gamma(t)$ consists of a finite number of mutually disjoint analytic Jordan curves. Clearly

$$
\int_{\Gamma(t)} d \theta=2 \pi
$$

In the covering surface $(W, \widehat{\mathbf{C}}, \pi)$ we consider a (pseudo-)metric on $W$ induced from the spherical metric on $\widehat{\mathbf{C}}$ by the projection $\pi$. The area of a set $G \subset W$ is denoted by $|G|$ and the length of a curve $\gamma \subset W$ is also denoted by $|\gamma|$ in the above induced metric. Put $A(t):=|R(t)|$, i.e. the area of $R(t)$. By denoting $\pi^{\prime}(z)=d \pi(z) / d z$, we have

$$
\begin{aligned}
A(t) & =\iint_{R(t)}\left(\frac{\left|\pi^{\prime}(z)\right|}{1+|\pi(z)|^{2}}\right)^{2} r d r d \theta \\
& =\int_{0}^{t}\left(\int_{\Gamma(r)}\left(\frac{\left|\pi^{\prime}(z)\right|}{1+|\pi(z)|^{2}}\right)^{2} r d \theta\right) d r \quad\left(z=r e^{\sqrt{-1} \theta}\right) .
\end{aligned}
$$

Clearly $t \mapsto A(t)$ is continuous and increasing, and

$$
\begin{aligned}
A(\infty) & :=\iint_{W}\left(\frac{\left|\pi^{\prime}(z)\right|}{1+|\pi(z)|^{2}}\right)^{2} r d r d \theta \\
& =\mu_{W} \iint_{F_{0}}\left(\frac{\left|\pi^{\prime}(z)\right|}{1+|\pi(z)|^{2}}\right)^{2} r d r d \theta=+\infty .
\end{aligned}
$$

Hence we conclude that

$$
\lim _{t \uparrow \infty} A(t)=A(\infty)=+\infty .
$$

Put $L(t):=|\Gamma(t)|$, the length of $\Gamma(t)=\partial R(t)$, so that

$$
L(t)=\int_{\Gamma(t)} \frac{\left|\pi^{\prime}(z)\right|}{1+|\pi(z)|^{2}} t d \theta \quad\left(z=r e^{\sqrt{-1} \theta}\right),
$$


which is also continuous in $t$. Denoting by $A^{\prime}(t)=d A(t) / d t$, we have

$$
A^{\prime}(t)=\int_{\Gamma(t)}\left(\frac{\left|\pi^{\prime}(z)\right|}{1+|\pi(z)|^{2}}\right)^{2} t d \theta .
$$

By the Schwarz inequality we see that

$$
\begin{aligned}
L(t)^{2} & =\left(\int_{\Gamma(t)} \frac{\left|\pi^{\prime}(z)\right|}{1+|\pi(z)|^{2}} t d \theta\right)^{2} \\
& \leq\left(\int_{\Gamma(t)}\left(\frac{\left|\pi^{\prime}(z)\right|}{1+|\pi(z)|^{2}}\right)^{2} t d \theta\right) \cdot\left(\int_{\Gamma(t)} t d \theta\right)=2 \pi t A^{\prime}(t)
\end{aligned}
$$

and thus we have obtained the inequality

$$
L(t)^{2} \leq 2 \pi t A^{\prime}(t)
$$

Choose and then fix an arbitrary $1 / 2<\tau<1$. We consider

$$
\Xi(s):=\left\{t \in[s, \infty): L(t) \geq A(t)^{\tau}\right\},
$$

which is closed in $[s, \infty)$. Choose an $r_{0}>0$ such that $R\left(r_{0}\right)$ is a regular region containing $\bar{F}_{0}$ so that $\partial R\left(r_{0}\right)$ consists of at least four components. Suppose that a sequence $0<r_{0}<r_{1}<\cdots<r_{n-1}$ for an $n \in \mathbf{N}, n>1$, has been chosen such that $L\left(r_{k}\right)<A\left(r_{k}\right)^{\tau}$ and $R\left(r_{k}\right)$ is regular for $1 \leq k \leq n-1$. Take an $s_{n} \in\left(\max \left\{n, r_{n-1}\right\}, \infty\right)$. Considering the logarithmic length

$$
\mathcal{L}(X):=\int_{X} \frac{d t}{t}
$$

for measurable subsets $X \subset(0, \infty)$, we observe that

$$
\mathcal{L}\left(\left[s_{n}, \infty\right)\right)=\int_{s_{n}}^{\infty} \frac{d t}{t}=[\log t]_{s_{n}}^{\infty}=+\infty .
$$

Using $1 / t \leq 2 \pi A^{\prime}(t) / L(t)^{2}$ for $t \in\left[s_{n}, \infty\right)$ as a consequence of (12) and $2 \pi A^{\prime}(t) / L(t)^{2} \leq 2 \pi A^{\prime}(t) / A(t)^{2 \tau}$ for $t \in \Xi\left(s_{n}\right)$, we deduce

$$
\begin{aligned}
\mathcal{L}\left(\Xi\left(s_{n}\right)\right) & =\int_{\Xi\left(s_{n}\right)} \frac{d t}{t} \leq 2 \pi \int_{\Xi\left(s_{n}\right)} \frac{A^{\prime}(t)}{A(t)^{2 \tau}} d t \\
& \leq 2 \pi \int_{s_{n}}^{\infty} \frac{A^{\prime}(t)}{A(t)^{2 \tau}} d t=2 \pi \int_{A\left(s_{n}\right)}^{A(\infty)} \frac{d A}{A^{2 \tau}} \\
& =2 \pi\left[\frac{1}{1-2 \tau} A^{-2 \tau+1}\right]_{A\left(s_{n}\right)}^{\infty}=\frac{2 \pi}{2 \tau-1} A\left(s_{n}\right)^{-2 \tau+1}<\infty .
\end{aligned}
$$


Hence $\mathcal{L}\left(\left[s_{n}, \infty\right) \backslash \Xi\left(s_{n}\right)\right)=\infty-(2 \pi /(2 \tau-1)) A\left(s_{n}\right)^{-2 \tau+1}=+\infty$. This shows that the set $\left[s_{n}, \infty\right) \backslash \Xi\left(s_{n}\right) \neq \emptyset$ is open and a fortiori we can find an $r_{n} \in\left(s_{n}, \infty\right)$ with $r_{n} \notin \Xi\left(s_{n}\right)$, i.e. $L\left(r_{n}\right)<A\left(r_{n}\right)^{\tau}$, such that $R\left(r_{n}\right)$ is regular. Since $r_{n}>s_{n}>\max \left\{n, r_{n-1}\right\}$, we have

$$
0<r_{0}<r_{1}<r_{2}<\cdots<r_{n-1}<r_{n} \uparrow \infty .
$$

Using this sequence we finally define

$$
R_{n}:=R\left(r_{n}\right), \quad \Gamma_{n}=\Gamma\left(r_{n}\right)=\partial R\left(r_{n}\right) \quad(n \geq 0) .
$$

Then $\left(R_{n}\right)_{n \geq 0}$ is a regular exhaustion of $W$ and

$$
\left|\Gamma_{n}\right| /\left|R_{n}\right|=L\left(r_{n}\right) / A\left(r_{n}\right)<A\left(r_{n}\right)^{\tau} / A\left(r_{n}\right)=A\left(r_{n}\right)^{\tau-1} \downarrow 0 \quad(n \uparrow \infty) .
$$

Thus the boundaries $\Gamma_{n}$ of the exhausting regions $R_{n}$ of $W$ are relatively short in the sense that

$$
\lim _{n \rightarrow \infty} \frac{\left|\Gamma_{n}\right|}{\left|R_{n}\right|}=0 .
$$

\subsection{Exhausting regions with long boundaries}

As a consequence of $W:=W[\sigma] \in O_{G}$, we have seen in the preceding Subsection 4.2 the existence of a regular exhaustion $\left(R_{n}\right)_{n \geq 0}$ of $W$ given in (13) that the boundaries $\Gamma_{n}=\partial R_{n}$ of $R_{n}$ are so short as to satisfy (14). We will see from a different view point that the same exhaustion $\left(R_{n}\right)_{n \geq 0}$ satisfies

$$
\liminf _{n \rightarrow \infty} \frac{\left|\Gamma_{n}\right|}{\left|R_{n}\right|}>0
$$

as a consequence of $W \in O_{G}$, which contradicts (14). Thus we should not have assumed that $W \in O_{G}$ and the proof of $W:=W[\sigma] \notin O_{G}$ will be over. The proof of (15) goes as follows.

Besides the main covering surface $(W, \widehat{\mathbf{C}}, \pi)$ we have two other covering surfaces $\left(W, F, \pi_{1}\right)$ and $\left(F, \widehat{\mathbf{C}}, \pi_{2}\right)$ involved in $(W, \widehat{\mathbf{C}}, \pi)$ with the relation $\pi=\pi_{2} \circ \pi_{1}$, where $F$ is the closed surface of genus 2 obtained from $F_{0}$ by pasting crosswise along $I_{01}$ and $I_{0,-1}$ and also along $I_{02}$ and $I_{0,-2}$. The metric on $F$ is the one induced from the spherical metric on $\widehat{\mathbf{C}}$ by $\pi_{2}$ and that on $W$ is the one induced from that on $F$ by $\pi_{1}$, which is identical with the one induced from the spherical metric on $\widehat{\mathbf{C}}$ by $\pi$ already considered in 
the preceding Subsection 4.2. The area (length, resp.) of a set $G$ (an arc $\gamma$, resp.) is denoted as before by the same notation $|G|(|\gamma|$, resp.) on either one of three surfaces $\left(F, \widehat{\mathbf{C}}, \pi_{2}\right),\left(W, F, \pi_{1}\right)$, and $(W, \widehat{\mathbf{C}}, \pi)$.

We denote by $\Phi(W)(\Phi(F)$, resp.) the set of closed curves $\varphi$ on $W(F$, resp.) which are not of homotop null. We maintain

$$
a(W):=\inf _{\varphi \in \Phi(W)}|\varphi|>0
$$

For a proof observe first that $\left(W, F, \pi_{1}\right)$ is smooth and complete (so that regular) and therefore the monodromy theorem assures that $\pi_{1}(\varphi) \in \Phi(F)$ if and only if $\varphi \in \Phi(W): \pi_{1}(\Phi(W)=\Phi(F)$. Since the metric on $W$ may be viewed as induced from one on $F$ by $\pi_{1}$, we see that $|\varphi| \geq\left|\pi_{1}(\varphi)\right|$. Hence

$$
a(W)=\inf _{\varphi \in \Phi(W)}|\varphi| \geq \inf _{\varphi \in \Phi(W)}\left|\pi_{1}(\varphi)\right|=\inf _{\varphi \in \pi_{1}(\Phi(W))}|\varphi|=\inf _{\varphi \in \Phi(F)}|\varphi|
$$

by which we only have to prove

$$
a(F):=\inf _{\varphi \in \Phi(F)}|\varphi|>0
$$

to conclude (16), i.e. $a(W) \geq a(F)>0$. Fix an arbitrary $p \in F$ and denote by $\Phi_{p}(F)$ the set of $\varphi \in \Phi(F)$ passing through $p$ and consider

$$
f(p):=\inf _{\varphi \in \Phi_{p}(F)}|\varphi| .
$$

Then $a(F)=\inf _{p \in F} f(p)$. We maintain that $f(p)>0$. Contrary to the assertion assume that $f(p)=0$ for a $p \in F$. Choose an open parametric disc $B$ centered at $p$ and let $\varepsilon$ be the distance between $p$ and $F \backslash B$ measured by the induced spherical metric on $F$. Then $f(p)=0$ implies that there is a $\varphi_{0} \in \Phi_{p}(F)$ such that $\left|\varphi_{0}\right|<\varepsilon$ so that $\varphi_{0} \subset B$, which is however impossible since $B$ is simply connected and $\varphi_{0}$ is not homotop null. Otherwise every $\varphi \in \Phi_{p}(F)$ is not contained in $B$, i.e. $\varphi \cap(F \backslash B) \neq \emptyset$, and a fortiori $|\varphi| \geq \varepsilon$, which leads to $f(p)=\inf _{\varphi \in \Phi_{p}(F)}|\varphi| \geq \varepsilon$, contradicting the present erroneous assumption $f(p)=0$. Thus we have seen that $f(p)>0$ for every $p \in F$. Next choose an arbitrary pair $(p, q)$ of points in $F$ and an arc $\gamma \subset F$ connecting $p$ and $q$. For any $\varphi \in \Phi_{q}(F)$, we have $\gamma \varphi \gamma^{-1} \in \Phi_{p}(F)$ and $\left|\gamma \varphi \gamma^{-1}\right| \leq|\varphi|+2|\gamma|$. Hence $f(q) \leq f(p)+2 d_{F}(p, q)$, which implies the (Lipschitz) continuity of $f$ on $F$, i.e. $|f(p)-f(q)| \leq 2 d_{F}(p, q)$ for every pair $(p, q)$ of points in $F$, where $d_{F}(p, q)$ is the induced spherical distance on $F$. Thus $f \in C(F)$ and $f>0$ on $F$ implies that $\inf _{F} f>0$, which proves (17). 
Concerning the particular exhaustion $\left(R_{n}\right)_{n \geq 0}$ introduced in the preceding Subsection 4.2, we suppose the relative boundary $\partial R_{n}=: \Gamma_{n}$ consists of a finite number $\nu_{n}$ of components $\Gamma_{n i}\left(1 \leq i \leq \nu_{n}\right): \Gamma_{n}=\bigcup_{1 \leq i \leq \nu_{n}} \Gamma_{n i}$. In view of the choice of $R_{0}=R\left(r_{0}\right)$ we have $\nu_{n} \geq 4(n \in \mathbf{N})$. Observe that $\Gamma_{n i}$ is not homologue null. Otherwise $\Gamma_{n i}$ is the boundary of a relatively closed region not containing $a$, which we denote by $\left(\Gamma_{n i}\right)$. Since $h=r_{n}$ on $\partial\left(\Gamma_{n i}\right)=\Gamma_{n i}$, the maximum principle yields $h \equiv r_{n}$ on $\left(\Gamma_{n i}\right)$ and thus $h$ must be a constant $r_{n}$ on $W$, which is clearly a contradiction. Since the homology group $\mathcal{H}(W)$ of $W$ is isomorphic to the factor group $\mathcal{F}(\mathcal{W}) / \mathcal{F}^{(\infty)}(\mathcal{W})$ of the homotopy group $\mathcal{F}(\mathcal{W})$ of $W$ by its commutator subgroup $\mathcal{F}^{(\infty)}(\mathcal{W})$, being homotop null implies being homologue null for any closed curve on $W$ and thus we see that $\Gamma_{n i} \in \Phi(W)\left(1 \leq i \leq \nu_{n}\right)$ so that $\left|\Gamma_{n}\right|=\sum_{1 \leq i \leq \nu_{n}} \Gamma_{n i} \geq \sum_{1 \leq i \leq \nu_{n}} a(W)$, i.e.

$$
\left|\Gamma_{n}\right| \geq \nu_{n} a(W) \quad(n \in \mathbf{N}) .
$$

We denote by $\rho\left(R_{n}\right)\left(\rho(F)\right.$, resp.) the Euler characteristic of $R_{n}(F$, resp.) multiplied by -1 . As a relatively compact subsurface of the planar surface $W, R_{n}$ is a planar surface bounded by $\nu_{n}$ Jordan curves $\Gamma_{n i}$ and a fortiori $\rho\left(R_{n}\right)=\nu_{n}-2$. Since the genus $g(F)$ of $F$ is 2 , i.e. $g(F)=2$, we see that $\rho(F)=2 g(F)-2=2$. Recall the main theorem of the Ahlfors theory of covering surfaces (cf. [1], see also e.g. [7], [10]) applied to the covering surface $\left(R_{n}, F, \pi_{1}\right)$ :

$$
\max \left\{\rho\left(R_{n}\right), 0\right\} \geq \rho(F) \frac{\left|R_{n}\right|}{|F|}-h_{F}\left|\Gamma_{n}\right|,
$$

where $h_{F}$ is a positive number depending only upon $F$ and independent of $R_{n}$. The inequality (18) implies $\left|\Gamma_{n}\right| / a(W) \geq \nu_{n}$ and the relation $\rho\left(R_{n}\right)=$ $\nu_{n}-2$ yields $\nu_{n}>\rho\left(R_{n}\right)$. Hemce $\left|\Gamma_{n}\right| / a(W)>\rho\left(R_{n}\right)$ holds. These with $\rho(F)=2$ transform (19) into the following form:

$$
\left|\Gamma_{n}\right| / a(W)>2\left|R_{n}\right| /|F|-h_{F}\left|\Gamma_{n}\right| .
$$

Rewriting the above inequality with $b:=2 a(W) /|F|\left(a(W) h_{F}+1\right)>0$, we obtain the inequality $\left|\Gamma_{n}\right| /\left|R_{n}\right|>b>0$ for every $n \in \mathbf{N}$ and thus we can finally deduce (15): $\liminf _{n \rightarrow \infty}\left|\Gamma_{n}\right| /\left|R_{n}\right| \geq b>0$. 


\section{REFERENCES}

[1] L. V. Ahlfors, Zur Theorie der Überlagerungsflächen, Acta Math., 65 (1935), $157-194$.

[2] L. V. Ahlfors and L. Sario, Riemann Surfaces, Princeton, 1960.

[3] O. Lehto and K. I. Virtanen, Quasikonforme Abbildungen, Springer, 1965.

[4] M. Nakai, On Evans potential, Proc. Japan Acad., 38 (1962), 624-629.

[5] M. Nakai and S. Segawa, Types of covering surfces, Suuriken-Kokyuroku, 1277 (2002), 84-93.

[6] M. Nakai and S. Segawa, A role of the completeness in the type problem for infinitely sheeted planes, Complex Variables, 49 (2004), 229-240.

[7] R. Nevanlinna, Analytic Functions, Springer, 1970.

[8] B. Rodin and L. Sario, Principal Functions, Van Nostrand, 1970.

[9] L. Sario and M. Nakai, Classification Theory of Riemann Surfaces, 1970.

[10] L. Sario and K. Noshiro, Value Distribution Theory, Van Nostrand, 1966.

[11] M. Tsuji, Potential Theory in Modern Function Theory, Maruzen, 1959.

Department of Mathematics

Nagoya Institute of Technology

Gokiso, Showa

Nagoya, 466-8555

Japan

Mailing Address:

52 Eguchi, Hinaga

Chita, 478-0041

Japan

nakai@daido-it.ac.jp 